\title{
Pitfalls in sentinel lymph node biopsy
}

\author{
Takao Yokoe
}

Published online: 30 September 2009

(C) The Japanese Breast Cancer Society 2009

Sentinel lymph node (SLN) biopsy is a common procedure to avoid unnecessary axillary lymph node dissection in breast cancer patients with negative nodes. However, there are some problems with SLN biopsy. One is the identification rate of SLNs. Many reports showed that the rate is higher with the combined dye and gamma probe-guided method than with the dye-guided method alone. Nevertheless, with the combined method, around 5\% of SLNs cannot be identified. In this issue, Ando et al. studied the axillary arch consisting of a muscular anomaly. They reported the usefulness of multidetector row computed tomography (MDCT) for an anatomical diagnosis of the axillary arch that affected SLD identification. Rokutanda et al. also reported a case of retromammary lymph node metastasis with a false-negative SLN biopsy. We have to keep in mind these anatomical varieties for SLN biopsy. The other important point is the existence of false-negative SLNs. Takei et al. gave us one of the answers to this problem. They showed that an intraoperative false-negative SLN biopsy did not affect the recurrence-free survival rate of breast cancer patients. They also showed the necessity of additional axillary radiotherapy or adjuvant systemic therapy. Nakashima et al. showed that abnormal accumulation of the radiotracer close to the radioactive spot indicates lymph node metastasis surrounding radioactive SLNs. They recommended additional lymph node biopsy surrounding radioactive SLNs.

We have to continue searching for the best method for SLN biopsies for patients with breast cancer.

T. Yokoe $(\square)$

Department of Surgery, Shibukawa General Hospital,

1338-4, Shibukawa, Gunma 377-0008,

Japan

e-mail: takayok@sougoubyouin.shibukawa.gunma.jp 